\title{
Segregation of "isotope" particles within colloidal molecules
}

\section{Citation}

Perry, Rebecca W., and Vinothan N. Manoharan. 2016. Segregation of 'isotope' Particles Within Colloidal Molecules. Soft Matter 12, no. 11: 2868-2876. doi:10.1039/c5sm02851e.

\section{Published Version}

10.1039/c5sm02851e

\section{Permanent link}

http://nrs.harvard.edu/urn-3:HUL.InstRepos:29997519

\section{Terms of Use}

This article was downloaded from Harvard University's DASH repository, and is made available under the terms and conditions applicable to Open Access Policy Articles, as set forth at http:// nrs.harvard.edu/urn-3:HUL.InstRepos:dash.current.terms-of-use\#OAP

\section{Share Your Story}

The Harvard community has made this article openly available.

Please share how this access benefits you. Submit a story.

Accessibility 
This is the accepted version of

Perry and Manoharan, Soft Matter 12(11): 2868-2876 (2016). doi: 10.1039/C5SM02851E

\title{
Segregation of "Isotope" Particles within Colloidal Molecules ${ }^{\dagger}$
}

\begin{abstract}
Rebecca W. Perry ${ }^{a}$ and Vinothan N. Manoharan ${ }^{a, b \neq}$
Clusters of spherical particles are called "colloidal molecules" because they adopt structures that resemble those of true molecules. In this analogy, the particles are the atoms, the attractive interactions between them are bonds, and the different structures that appear in equilibrium are isomers. We take this analogy a step further by doping colloidal molecules with colloidal "isotopes," particles that have the same size but different bonding energies from the other particles in the system. Our molecules are two-dimensional clusters consisting of polystyrene and silica microspheres held together by depletion interactions. Using a combination of optical microscopy and particle tracking, we examine an ensemble of 4- and 5-particle molecules at different isotope ratios. We find that the isotopes tend to segregate to particular positions in the various isomers. We explain these findings using a statistical mechanical model that accounts for the rotational entropy of the isomers and the different interaction potentials between the different types of particles. The model shows how to optimize the yield of any particular isomer, so as to put the isotopes in desired locations. Our experiments and models show that even in systems of particles with isotropic interactions, the structures of self-assembled molecules can be controlled to a surprisingly high extent through variables that are easily tuned in experiment.
\end{abstract}

\section{Introduction}

A "colloidal molecule" is a cluster of spherical colloidal particles that adopts the symmetry of a molecular structure. Since the term was first coined, ${ }^{1}$ the analogy between true molecules and colloidal molecules has been extended and deepened. The original, emulsion-based fabrication method ${ }^{2}$ and subsequent advances $^{3-9}$ aimed to develop colloidal molecules that could be used as building blocks for self-assembly of 3D macrostructures, and thus these methods focused on molecules whose structures do not change over time. Meanwhile, a parallel line of research has focused on understanding the self-assembly of individual colloidal particles into isolated molecules. ${ }^{10-17}$ In most of these studies, the interaction between the particles is weak-a few times the thermal energy $k_{B} T$-and consequently the structures can fluctuate over time. The distribution of structures can be predicted using a classical, equilibrium statistical mechanical formalism based on that used for true molecules. ${ }^{10,17}$ Thus, in these equilibrium colloidal molecules we can think of the particles as atoms, the attractive interactions between them as bonds,

\footnotetext{
${ }^{a}$ Harvard John A. Paulson School of Engineering and Applied Sciences, Harvard University, Cambridge MA 02138, USA.

${ }^{b}$ Department of Physics, Harvard University, Cambridge MA 02138, USA.

$\dagger$ Electronic Supplementary Information (ESI) available: methods for sample preparation and image processing. See DOI: 10.1039/C5SM02851E

$\ddagger$ To whom correspondence should be addressed: vnm@seas.harvard.edu
}

and the different structures that appear in equilibrium as isomers.

Here we take this analogy a step further: we consider how "isotopes" are incorporated into equilibrium colloidal molecules. In an atomic system, isotopes (for example, carbon-12 and carbon13) have different masses and different bonding energies but maintain the same bond geometry. Similarly, in our colloidal system, isotopes are two different kinds of microspheres (silica and polystyrene) that have different masses (silica being twice as dense as polystyrene) and different bonding energies but the same sizes. In the presence of a depletion attraction, the particles attract one another to form two-dimensional (2D) colloidal molecules with networks of bonds composed of equilateral triangles (Figure 1). The reason we call these particles "isotopes" is that their identical sizes preserve the bond angles. If the two species had different sizes, the smaller species could be coordinated by more than 6 particles of the larger species, making it unlikely for the particles to form an equilaterial triangular bond network. Because our two types of particles have the same size, one can be substituted for another without changing the bond network, just as with true atomic isotopes.

We create simple molecules containing fewer than 6 particles by mixing together isotopes in various ratios and letting the molecules equilibrate. We explore how particles of the minority isotope, or "dopants," segregate in the molecules. We were originally motivated by the question of whether more massive particles 

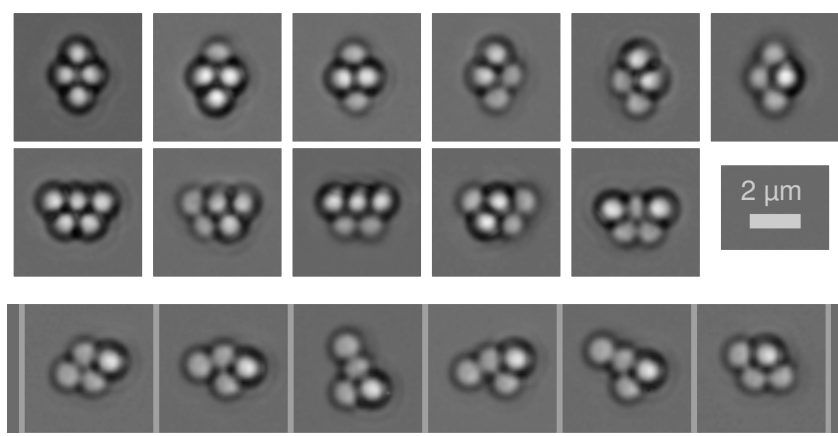

$t=0$

6.2
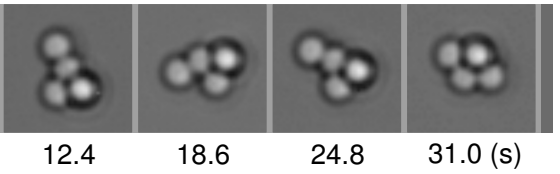

Fig. $11-\mu \mathrm{m}$-diameter polystyrene and silica microspheres form colloidal molecules. In these bright-field microscopy images, the polystyrene "isotopes" appear brighter than the silica ones (images in this figure are divided by a background and are gamma corrected with a value of 0.2 to enhance the distinction between isotopes). For a molecule with a particular structure, there are multiple isomers that differ in the number and positions of the isotopes (top and middle rows). Isomers that differ only in the position of the isotopes can equilibrate with one another through structural rearrangements (bottom row).

would preferentially migrate to the extremities of the molecule to maximize the rotational entropy, which is proportional to the moment of inertia. ${ }^{18}$ However, as was recently shown, ${ }^{19}$ any effect of mass on the rotational entropy should be exactly cancelled by compensating effects on the vibrational entropy.

Nonetheless, we do see that the isotopic dopants segregate to preferred positions of the colloidal molecules. We show how this segregation results from differences between the polystyrenepolystyrene, polystyrene-silica, and silica-silica bonds, along with entropic effects related to permutations of particles. Using a theoretical statistical mechanical model calibrated by the experimental data, we show how one might maximize the yield of any particular isomer-with the dopants appearing in particular positions-by varying the interparticle potentials and the stoichiometric ratio of the two isotopes.

These design rules, once validated by further experiments, might prove useful for the fabrication of "patchy particles," or colloidal structures with anisotropic interactions. For example, if the dopants were to carry specific functional groups, one could self-assemble molecules with the dopants in certain positions, and these molecules would themselves be patchy. Unlike methods of making patchy particles that use synthesis to control geometry, ${ }^{6,20}$ here the patchiness is emergent: even though all of the interactions are isotropic, minimization of the free energy leads to anisotropic clusters. A similar idea was proposed by Grünwald and Geissler ${ }^{21}$ for bidisperse spheres. We show that both entropic and energetic effects are important for controlling which isomers self-assemble in equilibrium, and we demonstrate the extent to which one can control the distribution of isomers.

\section{Materials and methods}

In our experiments, we prepare equilibrium ensembles of thousands of colloidal molecules, image them by raster-scanning, and then post-process the images to determine the molecules' sizes, compositions, and configurations.

\subsection{Making an ensemble of colloidal molecules}

To prepare the ensemble of molecules, we first construct a thin sample chamber in which colloidal particles can self-assemble into 2D molecules at the bottom of the chamber through diffusion and sedimentation. The sample chamber consists of two plasma-cleaned coverslips separated by $35-\mu \mathrm{m}$ Mylar ${ }^{\circledR}$ A spacers (sample chamber preparation protocol is provided in supplemental information). We fill the chamber with an aqueous colloidal suspension of polystyrene microspheres $(1-\mu \mathrm{m}$-diameter sulfate latex, Molecular Probes ${ }^{\circledR}$ by Life Technologies, lot \#1255616, batch \#1169661, used as received) and silica microspheres (1$\mu$ m-diameter 8000 Series Silica Particle Size Standards, Duke Standards from Thermo Scientific, lot \#41291, batch \#8100-013, used as received) in $45 \mathrm{mM}$ sodium dodecyl sulfate (SDS) solution. The total microsphere volume fraction is $5 \times 10^{-5}$ which, after sedimentation in our chambers, yields an area fraction of $2 \times 10^{-3}$.

The SDS micelles create a depletion force between particles and between particles and the coverslip ${ }^{17}$, allowing the particles to form 2D molecules. After the microspheres reach the bottom of the chamber, they diffuse along the coverslip. Rarely do we see them leave the plane. When two microspheres encounter one another, they also experience a depletion attraction, but because this attraction is weaker than that between the particles and coverslip, ${ }^{22,23}$ they can bind and unbind. As a result, molecules can rearrange between different isomers. Isomers persist for minutes before rearrangements occur on the order of ten seconds (Figure 1).

Over the course of an hour-long assembly and equilibration period, the silica and polystyrene isotopes form an ensemble of colloidal molecules, all of which are subunits of a triangular lattice. The silica and polystyrene isotopes can assemble in this way because they have nearly the same size: $1.0 \mu \mathrm{m}$ diameter, as measured from the lattice spacing in pure crystals of each. Particles detach from the molecules infrequently compared to how often they rearrange within a molecule. Molecules also merge together infrequently because the density of microspheres on the surface is low. We therefore assume each molecule to be in equilibrium and independent of its neighbors.

\subsection{Imaging and post-processing}

To image the molecules, we use a Nikon Ti-E inverted microscope with a $60 \times$ water-immersion objective, a $1.5 \times$ tube lens, and a modified stage that allows micrometer-scale control over the in-plane position of the sample. To modify the stage, we remove the stage handle and then mount below the stage two vernier micrometer heads (Newport SM-25 with mounting hardware Newport $A B-3$ ) at orthogonal directions and aligned with actuator push blocks (Newport AB-4) also mounted below the stage. We use rubber bands to keep tension between the mounting hardware holding each micrometer head and the actuator push blocks. With these micrometer heads, we manually move the sample in a raster-scan pattern and capture images on a Photonfocus MV-D1024E-C021-160-CL-14 monochrome CMOS camera with a CameraLink cable connected to an EPIX PIXCI E8 frame 


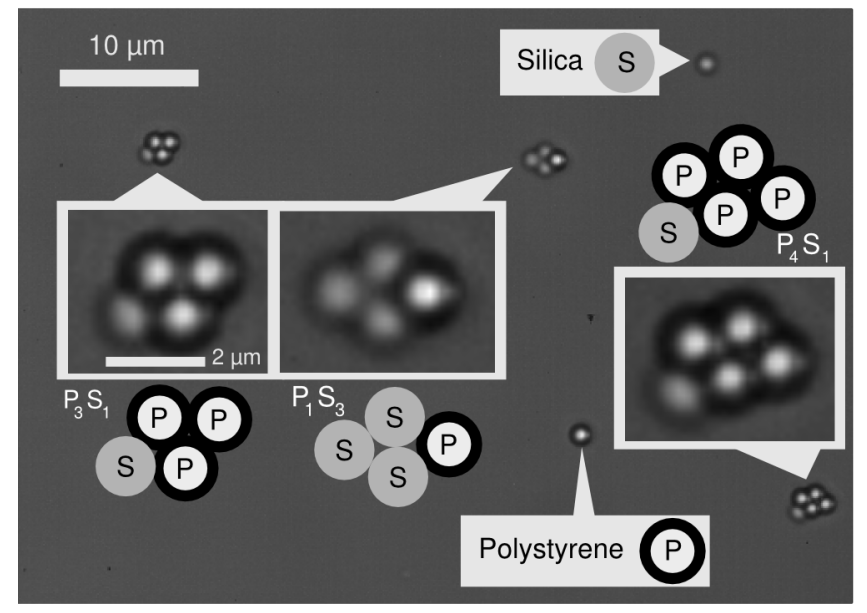

Fig. 2 Optically distinguishing silica and polystyrene particles. Owing to their different indices of refraction, the polystyrene $(P)$ and silica $(S)$ particles can be distinguished in bright-field optical micrographs. The polystyrene particles are bright and surrounded by dark rings whereas the silica particles are dimmer. This section of a micrograph shows a single sphere of each isotope, two 4-particle molecules with different compositions, and a 5-particle molecule. Each $\mathrm{N}$-particle molecule is labeled as $\mathrm{P}_{X} \mathrm{~S}_{N-X}$, denoting its composition. The insets are magnified by a factor of approximately 3.5 .

grabber. Each image is of an area approximately $120 \times 120 \mu \mathrm{m}$ in size. We move the stage in $150 \mu \mathrm{m}$ increments to leave a border between frames, ensuring molecules are not double-counted as they diffuse along the coverslip. After each translation of the stage, we adjust the focus so that silica and polystyrene particles are distinguishable by eye, and then we capture an image (Figure 2).

Repeating this process, we gather 1,765 images across 4 samples with different stoichiometric ratios of polystyrene (P) and silica (S). By computationally analyzing these images, we identify and classify 1,685 rigid 4-particle molecules and 1,434 rigid 5 -particle molecules. We do not attempt to follow individual molecules over time. Instead, our analysis is based on ensemble averages.

Our post-processing routine locates the individual particles, determines the type of each particle, groups the particles into molecules using a cutoff distance to distinguish bound and unbound particles, and categorizes each molecule as a specific isomer. We use Trackpy, ${ }^{24}$ an open-source software package based on the particle-tracking algorithms of Crocker and Grier, ${ }^{25}$ to locate and characterize the particles, and we use custom algorithms for subsequent particle and molecule classification (see supplementary information for more details).

\section{Results and Discussion}

\subsection{Position of isotopes in 4- and 5-particle molecules}

In even the smallest molecules, containing 4 particles, we find that certain isomers occur more frequently than others. 4-particle molecules containing three particles of one isotope and a single dopant of the other, $\mathrm{P}_{3} \mathrm{~S}_{1}$ and $\mathrm{P}_{1} \mathrm{~S}_{3}$, each have two isomers: the dopant can can be on the short axis or the long axis of the diamond-shaped ground state (Figure 3). Assuming all interac- tions are the same, one expects these two isomers to be found in equal amounts. However, we find that the silica dopants in the $\mathrm{P}_{3} \mathrm{~S}_{1}$ molecules are located on the long axis in $62.2 \%$ of the $\mathrm{P}_{3} \mathrm{~S}_{1}$ molecules. In contrast, we find that the polystyrene dopants in the $P_{1} S_{3}$ molecules are more often located on the short axis, but by a smaller margin.

The distribution of the other 4-particle isomers, which contain equal numbers of silica and polystyrene particles, also differs from the distribution expected when all interactions are the same. We describe the three $\mathrm{P}_{2} \mathrm{~S}_{2}$ isomers in terms of where the two silica particles are located: along the short axis, along the long axis, or along an edge. If all interactions were the same, we would expect the isomer with the silica particles along an edge to be the most common, because there are four different ways to place two indistinguishable particles along an edge. These four ways include two chiral enantiomers that we group together because they have identical bond networks. The other two isomers can be constructed in only one way (formally, the differences in the expected populations are related to the permutational—or, equivalently, rotational - entropy of the different isomers, as we discuss later). In our experiments, we see that the silica spheres do appear along an edge in $68.8 \% \approx 4 / 6$ of the molecules, as expected (Figure $3, \mathrm{P}_{2} \mathrm{~S}_{2}$ molecules). However, the populations of the two less common isomers show significant differences from the expected probabilities: the silica particles are twice as likely to be found on the long axis as on the short axis.

The rigid 5-particle trapezoidal molecules display 18 different isomers with 1 or 2 dopants each. Particles in the trapezoid can be located at the vertices of the acute angles ( 2 positions), the vertices of the obtuse angles ( 2 positions), or on the edge ( 1 position). The observed probabilities of the various isomers are not consistent with those predicted by a model that accounts only for the permutational entropy and that assumes the interactions have the same strength. We find that molecules with silica dopants on the edge are suppressed, while those with silica dopants at the vertices of the acute angles are enhanced. The opposite is true of polystyrene dopants.

\subsection{Statistical mechanical model}

To understand the observed probabilities of the different isomers, we construct a statistical mechanical model incorporating the effects of entropy as well as the interactions between pairs of bound spheres. As shown in previous work, ${ }^{17,26}$ when the interactions are short-ranged (as they are in our experimental system) one can use a "sticky sphere" approximation in which each interaction (S-S, P-S, P-P) is characterized by a single "sticky parameter" $(\kappa)$, which is a function of both the depth and curvature of the potential. We shall show that the probabilities we observe can be explained in terms of differences in sticky parameters. We show furthermore that whereas the permutational entropy cannot be changed in the experiments, the sticky parameter can be tuned to produce a high yield of desired isomers.

The equilibrium probability of observing a particular colloidal molecule is proportional to the configurational part of the classical canonical partition function: $P \propto Q$. As in statistical mechan- 

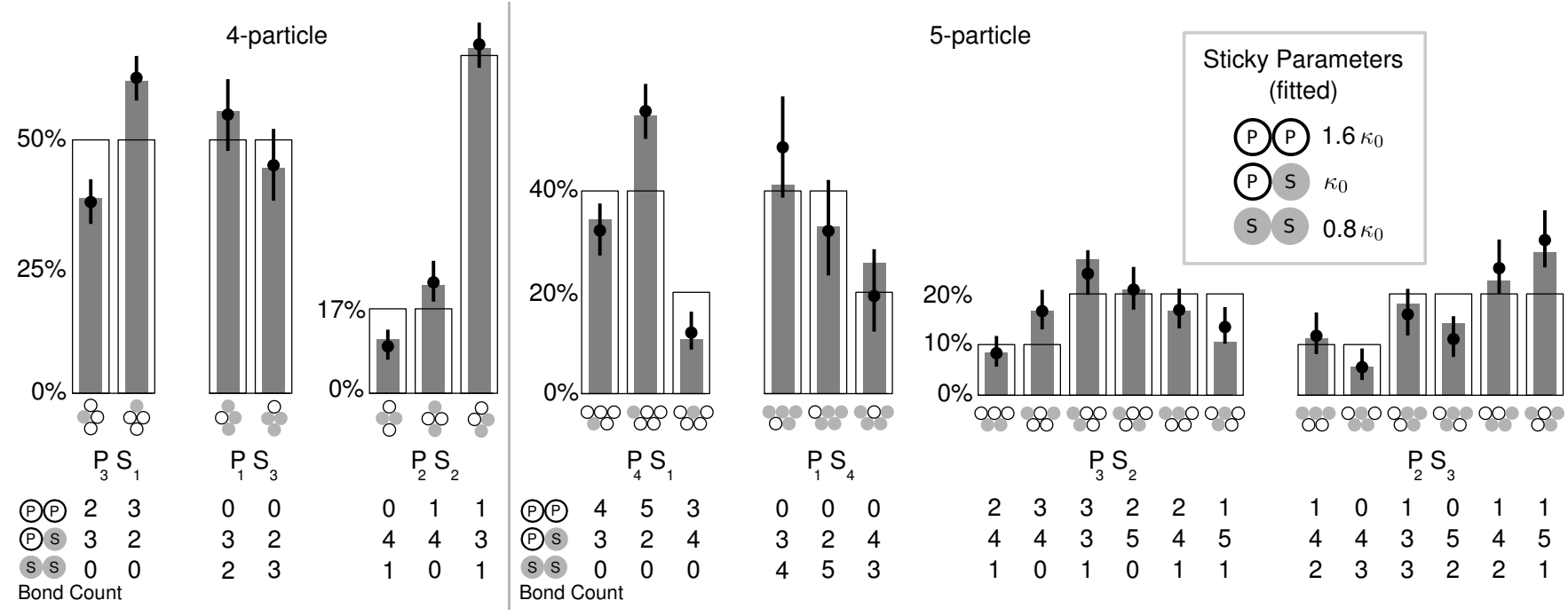

Fig. 3 Selective placement of two species of colloids in small colloidal molecules. The distribution of isomers in our experiments, shown as black points, is captured by a model, shown as gray bars, that includes permutational entropy and two ratios of sticky parameters (see text). The hollow bars show the expected probabilities with identical sticky parameters, in which case the distribution is determined entirely by the permutational entropy. The probabilities within each set of isomers (for example, $\mathrm{P}_{3} \mathrm{~S}_{1}$ ) sum to $100 \%$. The error bars are $95 \%$ confidence intervals.

ical treatments of true molecules, the partition function can be separated into translational, rotational, and vibrational components: 10

$$
Q=Q_{\text {trans }} Q_{\text {rot }} Q_{\text {vib }}
$$

The vibrational partition function depends on the potential energy of the molecule. We assume this energy is pairwise additive, and we expand the pair potential about its minimum, truncating the expansion at second order (a harmonic approximation). We can then express the partition function in terms of the sticky parameters $\kappa_{i}$ for each bond $i$ and $Q_{\text {vib }}^{\prime}$, the part of the vibrational partition function that does not depend on the magnitude of the spring constants:

$$
Q=Q_{\text {trans }} Q_{\text {rot }} Q_{\mathrm{vib}}^{\prime} \prod_{i=1}^{n} \kappa_{i}, \quad \text { with } \quad \kappa_{i}=\frac{e^{-\beta U_{0 i}}}{d \sqrt{\frac{2}{\pi} \beta U_{0}^{\prime \prime}}},
$$

where $\beta=1 / k_{B} T, U_{0 i}$ the depth of the potential well for bond $i, U_{0}^{\prime \prime}$ the curvature at the potential minimum, and $d$ the microsphere diameter.

In calculating the populations of different isomers, we need not calculate the translational component, $Q_{\text {trans }}$, because it is the same for all isomers of a given number of particles. The rotational partition function $Q_{\text {rot }}$, is proportional to the square root of the moment of inertia and the chirality $\chi(\chi=1$ for a chiral molecule and $\chi=2$ for an achiral one) and inversely proportional to the symmetry number $\sigma^{10,19}$. The product of $Q_{\mathrm{vib}}^{\prime}$ and the moment of inertia is also the same for any $N$-particle molecule, regardless of composition $^{19}$. Thus, ignoring prefactors that are constant for all molecules of a given $N$, we find that the probability of observing a particular $N$-particle molecule is:

$$
P \propto Q \propto \frac{\chi}{\sigma} \prod_{i=1}^{n} \kappa_{i}
$$

where $n$ is the number of bonds.

We determine the sticky parameters for the three different types of bonds (S-S, P-S, P-P) by first calculating $\chi / \sigma$ for each isomer and then fitting the model to the experimental data. To demonstrate how $\chi$ and $\sigma$ are determined for a heterogeneous molecule, we consider the set of 4-particle $\mathrm{P}_{2} \mathrm{~S}_{2}$ molecules. There are 4 ! ways of arranging 4 distinguishable particles into this 4 particle molecule, but its 2 -fold rotational axis means that only 12 of these configurations (or "colorings") are distinct (see Figure 4 and ref. ${ }^{19}$ ). We then subdivide the colorings into groups corresponding to the three isomers (including one isomer that is a chiral pair). To do this, we assign any two of the colors to represent silica (two shades of blue in Figure 4). This results in 8 permutations in which the pair of silica particles are along an edge, 2 permutations where they are on the long axis, and 2 permutations where they are on the short axis. These counts are proportional to the factor $\chi / \sigma$ for each isomer (along an edge: $\chi=2, \sigma=1$; along the long axis: $\chi=1, \sigma=2$; along the short axis: $\chi=1, \sigma=2)$. Alternatively, one can use the binomial coefficient instead of starting with the total number of colorings: there are $\left(\begin{array}{l}4 \\ 2\end{array}\right)=6$ ways to choose 2 of the 4 particles to be silica. These 6 permutations are then subdivided as before and yield a 4:1:1 ratio of the three isomers. The permutational part of the rotational partition function, $\chi / \sigma$, determines the probabilities represented by the empty bars in Figure 3 .

\subsection{Fitting the model to the data}

Although there are three sticky parameters in our experiments, P$\mathrm{P}, \mathrm{S}-\mathrm{S}$, and P-S, the probabilities are sensitive only to their ratios. We choose the P-P and S-S sticky parameters as our fit parameters, and we assign the P-S sticky parameter a value $\kappa_{0}$. Using only these two fitting parameters, we obtain an excellent fit to the data when P-P $=1.6 \kappa_{0}$ and S-S $=0.8 \kappa_{0}$. This range of sticky parameters is reasonable, as it corresponds to a difference of less 


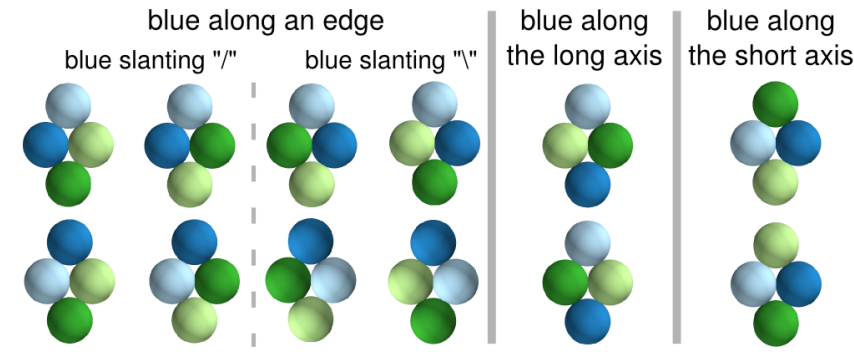

Fig. 4 Permutations of particles in a 4-particle heterogeneous molecule. The 4 ! colorings of a 4-particle molecule are reduced to these 12 colorings when rotation in the plane is allowed. The 12 colorings are then subdivided according to where the two blue particles are. This subdivision results in 8 permutations with the blue particles along an edge, 2 permutations with the blue particles on the long axis, and 2 permutations with the blue particles on the short axis, yielding the predicted 4:1:1 ratio of the three isomers of $P_{2} S_{2}$.

than $1 k_{B} T$ between the deepest and shallowest interaction potentials, assuming identical curvature $U_{0}^{\prime \prime}$. This fitting procedure shows that the polystyrene particles form stronger bonds than the silica particles do.

To independently verify the fitted values, we examine the homogeneous 3- and 4-particle molecules found in the same experimental data set, and we measure the absolute values of $\kappa$ for P-P and S-S bonds by comparing the number of rigid molecules to the numbers of excited-state molecules with fewer than $2 N-3$ bonds using the method of Holmes-Cerfon et al. ${ }^{26}$ which we previously used in 6-particle homogeneous clusters. ${ }^{17}$ We find that the P-P bonds have a sticky parameter of approximately 135, and the S-S bonds have a sticky parameter of approximately 50. The factor of 2.7 between these sticky parameters is close to the factor of 2 found by fitting the distributions. We take this as confirmation that the fitted relative P-P and S-S values are sensible, and we estimate that the P-S sticky parameter is between 60 and 85 .

Looking back at the data, we can see that the different stickiness values affect the probability distribution in a logical way. The isomers have different numbers of each type of bond ("Bond Count" in Figure 3), and the most frequently observed isomers have the strongly-binding polystyrene isotopes in locations where they can form the most bonds and the weakly-binding silica isotopes in locations where they can form the fewest.

By examining isomers containing a single dopant, we can verify that the magnitude of the sticky parameter for the crossinteractions (P-S) lies between those for the P-P and S-S interactions. In the $\mathrm{P}_{3} \mathrm{~S}_{1}$ molecules, the silica dopant is more frequently found with 2 bonds as opposed to 3 bonds. In other words, the P-P bond is preferred, and thus has larger $\kappa$ than the P-S bond. Using the same analysis on the $\mathrm{P}_{1} \mathrm{~S}_{3}$ molecules, we learn that the P-S bond is slightly stronger than the S-S bond. This qualitative ordering of the bonds from least-sticky to most-sticky (S-S, S-P, $\mathrm{P}-\mathrm{P}$ ) is consistent with the quantitative results of the fit.

The agreement between the data and the model shows that the distribution of isomers and the segregation of different isotopes can be explained in terms of the permutational entropy and the sticky parameters for each bond type. The masses of the dopants and the positions of these masses are not needed to account for the observed probabilities; that is, there is no evidence for a "mass effect"18,19. But why are the sticky parameters different for the three different bond types? After all, the depletion attraction should be the same between all isotopes. One possibility is that while the strength of the depletion attraction is the same, the electrostatic and van der Waals forces are different in the three interactions. For example, the silica particles could have a higher surface charge density and, therefore, a stronger repulsive contribution to the interaction potential as compared to the polystyrene particles. This would account for the smaller S-S sticky parameter.

\subsection{Maximizing the yield of a particular isomer relative to others of the same composition}

The variation in sticky parameters suggests that they can be controlled. Therefore, having shown the agreement between model and data, we now examine the question of how to tune the parameters to maximize the yield of certain clusters. In the $\mathrm{P}_{2} \mathrm{~S}_{2}$ molecules, the model shows us how to maximize the probabilities of any of the three isomers. For example, setting S-S to be strong and P-P to be weak preferentially locates the silica spheres on the long axis (as seen in our experiments), and setting P-P and S-S to be $4 \kappa_{0}$ should yield equal numbers of all three $\mathrm{P}_{2} \mathrm{~S}_{2}$ isomers.

The effect of the sticky parameters on the probability distributions raises the following question: What is the maximum deviation from the equal-interaction probability distribution that one could reasonably achieve in an experiment? The constraints are that all the bonds need to be strong enough to make a molecule that persists for long times, yet weak enough to allow rearrangements on an experimental timescale. Given that the transition rates scale inversely with $\kappa,{ }^{26}$ we can estimate that $\kappa$ could span a factor of 100 , perhaps from 40 to 4000 . For homogeneous molecules with $\kappa=40$ a transition would occur every minute or so, and for homogeneous molecules with $\kappa=4000$ a transition would occur every day or so.

Our model predicts that at large values of $\kappa$, the equilibrium probability of certain isomers can be increased to above $90 \%$. In Figure 5a, we choose S-S as the weakest interaction and P-P as the strongest, just as in the experiments. But we choose $\kappa_{\mathrm{P}-\mathrm{P}}$ one hundred times larger than $\kappa_{\mathrm{S}-\mathrm{S}}$. With these values the model predicts a distribution in which the contribution from permutational entropy is overwhelmed by that of the interaction potentials. The P-P bonds are so strongly favored that many of the preferred isomers show segregation between polystyrene and silica. One of the $\mathrm{P}_{3} \mathrm{~S}_{2}$ isomers (third from left) contains a polystyrene triangle but is suppressed. This is possible because the S-S bonds are much weaker than the P-S bonds.

When both types of self-self bonds are stickier than the crossinteractions, a different distribution of isomers might be stabilized (Figure 5b). However, by comparing the results in Figure 5a and $5 b$, we see that the probabilities of certain isomers cannot be significantly enhanced. For example, the distribution of $\mathrm{P}_{4} \mathrm{~S}_{1}$ and $\mathrm{P}_{1} \mathrm{~S}_{4}$ molecules, which each have three isomers but just two types of interactions, cannot be tuned so that any isomer is enhanced. Nonetheless, the model predicts that the yields of certain isomers, 


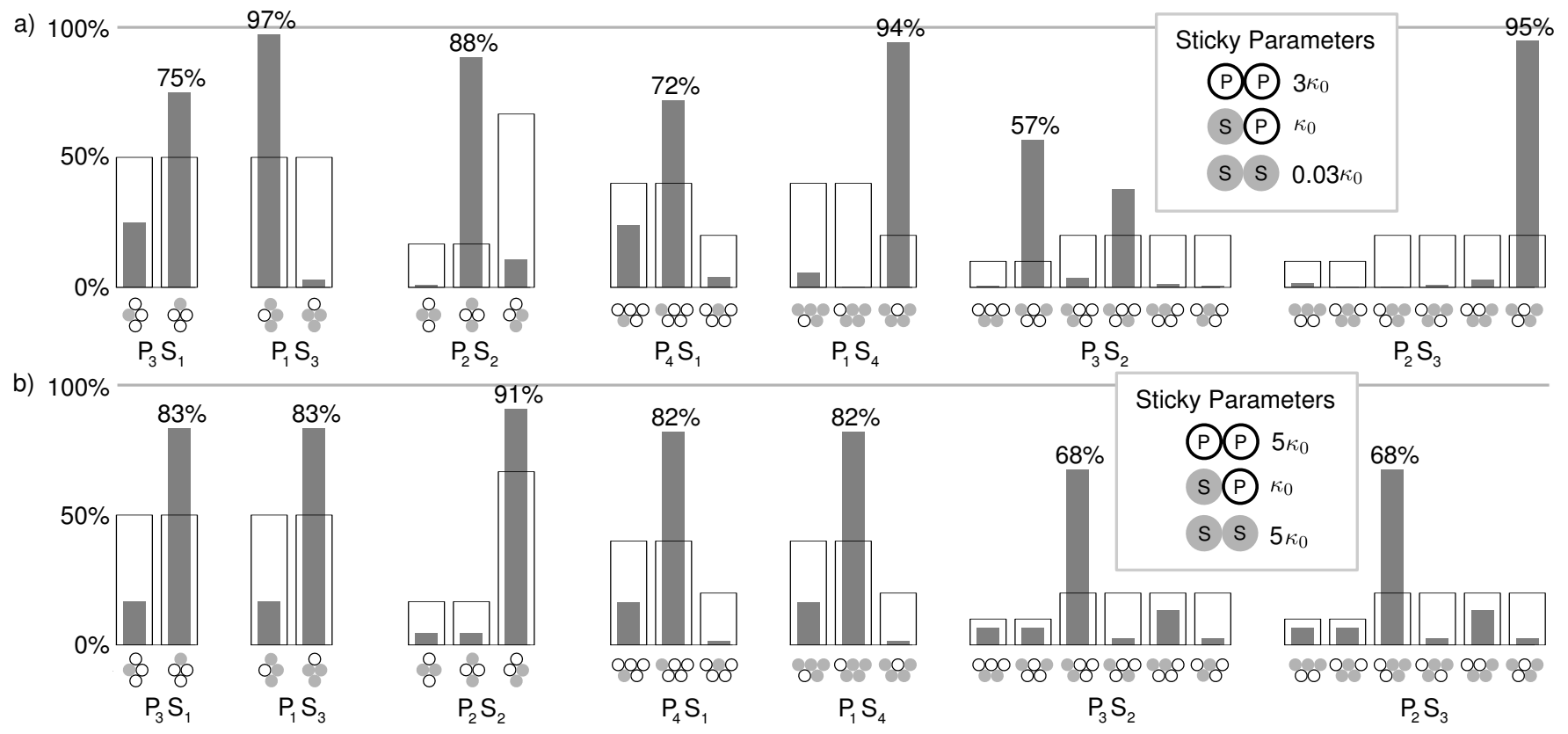

Fig. 5 Extrema in selectivity of colloidal molecules. Theoretical distributions of isomers with two extremal sets of sticky parameters. In both distributions, certain isomers are predicted to be enhanced above their equal-interaction probabilities by more than a factor of 3 . Hollow bars show the permutational-entropy-dominated distribution calculated for isotopes with identical sticky parameters, for comparison.

such as those $\mathrm{P}_{4} \mathrm{~S}_{1}$ and $\mathrm{P}_{1} \mathrm{~S}_{4}$ molecules with single dopants at the upper vertices of the trapezoid, can be optimized from $0.02 \%$ to $82 \%$ probability, and anywhere in between. In fact, when the P-P bonds are made 100 times stronger than the S-P bonds, the yield of this isomer approaches $99 \%$.

The values of $\kappa$ we use to achieve these high yields correspond to experimentally realizable potentials. To construct sticky parameters spanning two orders of magnitude as in Figure 5a, the depths of the interaction potentials need only have a difference of $\ln (100) k_{B} T \approx 4.6 k_{B} T$ between the weakest and strongest bonds (for example, $U_{0, \mathrm{~S}-\mathrm{S}}=-6.5 k_{B} T, U_{0, \mathrm{P}-\mathrm{S}}=-10 k_{B} T, U_{0, \mathrm{P}-\mathrm{P}}=$ $-11.1 k_{B} T$ ). This distribution of bond types could be achieved using DNA-mediated interactions. ${ }^{27}$

\subsection{Maximizing the yield of molecules with particular com- positions $\mathbf{P}_{X} \mathbf{S}_{N-X}$}

Having shown that the stickiness of the interparticle interactions leads to selective placement of dopants, we now turn to the problem of optimizing the yield of molecules with a specific composition: $\mathrm{P}_{X} \mathrm{~S}_{N-X}$. Combined, these two design criteria should allow for system-wide optimization of a specific isomer.

When we vary the polystyrene fraction in our experiments, we see that the distribution of compositions of both 4- and 5particle molecules $\left(\mathrm{P}_{X} \mathrm{~S}_{N-X}\right)$ shifts as the total polystyrene fraction increases (Figure 6). We calculate the polystyrene fraction, $\phi_{\mathrm{P}}$, by counting all of the particles participating in 4 and 5-particle molecules. The distribution of compositions is symmetric at $\phi_{\mathrm{P}}=0.50$ and shifts to heavily favor molecules with more polystyrene at $\phi_{\mathrm{P}}=0.73$ (Figure 6). The largest differences are at the extrema of these probability distributions: the percentage of molecules composed purely of silica progressively shrinks with each increase in $\phi_{\mathrm{P}}$, while the percentage of pure polystyrene molecules steadily increases to four times its value at $\phi_{\mathrm{P}}=0.50$. We see that even small changes in $\phi_{\mathrm{P}}$, such as going from 0.50 to 0.55 , can change the distributions significantly. In the 4-particle distribution, the $\mathrm{P}_{1} \mathrm{~S}_{3}$ and $\mathrm{P}_{3} \mathrm{~S}_{1}$ molecules have nearly equal probabilities at $\phi_{\mathrm{P}}=0.50$, but distinctly different ones at $\phi_{\mathrm{P}}=0.55$, and similarly for the $\mathrm{P}_{1} \mathrm{~S}_{4}$ and $\mathrm{P}_{4} \mathrm{~S}_{1}$ molecules.

Because these molecules are formed by random aggregation in a well-mixed pool of two types of particles, it is natural to model the distribution of compositions using the weighted binomial distribution. In our model, we take our measured 4- and 5 -particle polystyrene fraction, $\phi_{\mathrm{P}}$, as the probability that any one particle added to a cluster is polystyrene. The probability of a certain molecule composition $\mathrm{P}_{X} \mathrm{~S}_{N-X}$ occurring, from among all molecules of size $N$, is

$$
P\left(\mathrm{P}_{X} \mathrm{~S}_{N-X} \mid \phi_{P}\right)=\left(\begin{array}{l}
N \\
X
\end{array}\right) \phi_{\mathrm{P}}^{X}\left(1-\phi_{\mathrm{P}}\right)^{N-X}
$$

Note that in our system the number of molecules at a given $N$ changes over time as molecules coalesce. We find that many 4and 5-particle molecules can be observed within a couple of hours after sample preparation, but afterward the larger molecules dominate. However, one can grow the molecules in microwells ${ }^{10}$ to keep them from coalescing.

As shown in Figure 6, the weighted binomial distribution captures all the features of the data. The model predicts that it is possible to maximize any particular composition $\mathrm{P}_{X} \mathrm{~S}_{N-X}$ by setting $\phi_{\mathrm{P}}$ to $X / N$ (Figure 7). For example, to optimize $\mathrm{P}_{1} \mathrm{~S}_{3}$ molecules, one would use $\phi_{\mathrm{P}}=0.25$. This polystyrene fraction yields a population in which $42 \%$ of the 4-particle molecules are $\mathrm{P}_{1} \mathrm{~S}_{3}$. The closer the molecular composition is to $\mathrm{P}_{N / 2} \mathrm{~S}_{N / 2}$, and the larger the molecule, the smaller is the theoretical maximum yield. 


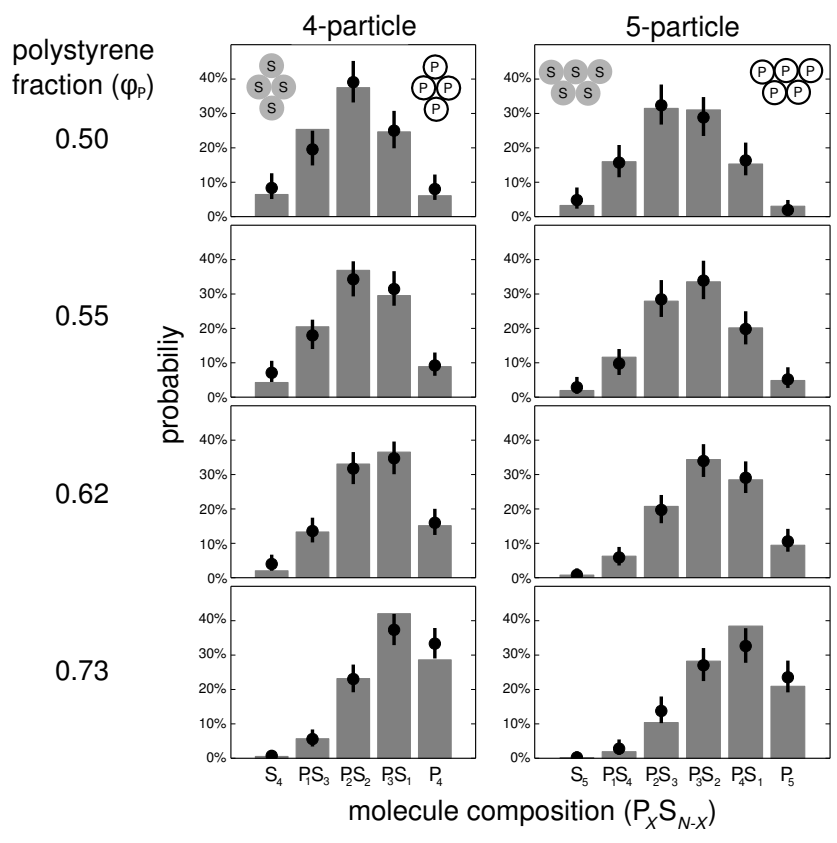

Fig. 6 Dependence of colloidal molecule composition on polystyrene fraction. The populations of $\mathrm{N}$-particle molecules consist of $N+1$ compositions ranging from pure silica to pure polystyrene. Experimental data on 4- and 5-particle molecules (black data points with 95\% confidence intervals) are modeled well by the weighted binomial distribution (gray bars).
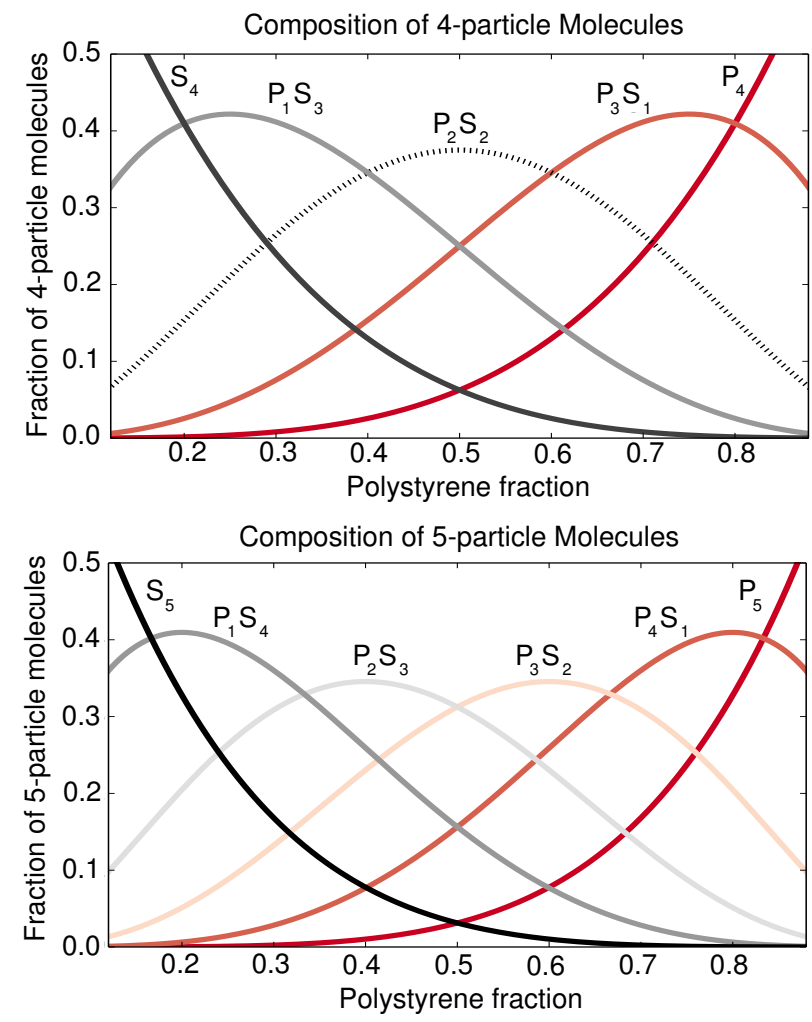

Fig. 7 The weighted binomial model as a function of polystyrene fraction. The weighted binomial distribution (Equation 4) predicts the population distribution as a function of the polystyrene fraction. Each composition has a single maximum. Any two compositions have equal probability at a single polystyrene fraction between 0 and $100 \%$, indicated by where the curves intersect.
It is perhaps surprising that this purely agglomerative model fits the data so well, given that it does not account for the sticky parameters of the particles. We would expect that the difference between the P-P and S-S sticky parameters would favor the incorporation of polystyrene into the molecules at the expense of silica. However, the discrepancy in sticky parameters also means that polystyrene spheres should be preferentially incorporated into larger clusters and silica particles into smaller ones. Because we measure the polystyrene fraction by counting only the 4and 5-particle molecules (on which we have complete data), the measured silica fraction $\left(1-\phi_{\mathrm{P}}\right)$ will be higher than that measured for all the molecules. This effect shifts the distribution of compositions back toward higher silica fractions through the term $\left(1-\phi_{\mathrm{P}}\right)^{N-X}$ in Equation 4 . Thus the error in neglecting the sticky parameters is compensated by the error in measuring the polystyrene fraction. In practice, the polystyrene fraction in the clusters must always be measured and cannot be assumed equal to that in the bulk, since the two species have different sedimentation rates. So, despite the compensating errors, we would argue that the model nonetheless gives useful predictions when the isotope fractions are measured using small clusters.

The binomial distribution shows where we can expect to generate equal numbers of molecules with different compositions. For example, one might want to generate singly-doped molecules of compositions $\mathrm{P}_{1} \mathrm{~S}_{N-1}$ and $\mathrm{P}_{N-1} \mathrm{~S}_{1}$. The optimal polystyrene fraction to obtain identical yields of $N$-particle molecules with compositions $\mathrm{P}_{X} \mathrm{~S}_{N-X}$ and $\mathrm{P}_{Y} \mathrm{~S}_{N-Y}$ is given by:

$$
\phi_{\mathrm{P}}=\frac{\left(\begin{array}{l}
N \\
X
\end{array}\right)^{\frac{1}{Y-X}}}{\left(\begin{array}{l}
N \\
X
\end{array}\right)^{\frac{1}{Y-X}}+\left(\begin{array}{l}
N \\
Y
\end{array}\right)^{\frac{1}{Y-X}}} .
$$

\subsection{Maximizing the yield of a particular isomer relative to all molecules}

With these design strategies in hand, we now return to our initial goal of maximizing the yield of any one particular isomer relative to all molecules, not just molecules of the same composition. For example, suppose we want to maximize the yield of the $\mathrm{P}_{1} \mathrm{~S}_{3}$ isomer in which the polystyrene sphere is on the short axis. We assume that the molecules cannot coalesce once formed, which would be the case if the experiment were done in microwells ${ }^{10}$. We have four parameters to control: the total particle concentration, the stoichiometric ratio, and the two ratios of sticky parameters. We would like $100 \%$ of the molecules to have four particles in them, but Poisson statistics tells us that for random loading the best we can do by simply modifying the total particle concentration is $19.5 \%$. We would like a large fraction of these wells that contain four particles to have one polystyrene particle and three silica particles, so we use a total polystyrene ratio of 0.25 (or a modification thereof to obtain $\phi_{\mathrm{P}}=0.25$ ), yielding $42 \%$ of the four-particle molecules with the correct composition. Finally, the interaction energies must be set so that the dopant prefers to sit on the short axis of the diamond-shaped molecule. Our statistical mechanical model suggests that we can achieve at least a $90 \%$ yield of $\mathrm{P}_{1} \mathrm{~S}_{3}$ molecules with the dopant in the right place. This reasoning suggests that we may be able to construct an equilib- 
rium system in which $19.5 \% \times 42 \% \times 90 \%=7.4 \%$ of the molecules are the exact isomer we desire. This is three times the prevalence that would result from equal interactions and equal numbers of polystyrene and silica. Much higher yields might be achieved if the number of particles per well can be controlled.

\section{Conclusions}

Our results show that it should be possible to control the distribution of self-assembled heterogeneous colloidal molecules through two mechanisms: by varying the interactions (more precisely, sticky parameter ratios) and by varying the stoichiometric ratio (polystyrene fraction). The models that describe our data well, Equations 3 and 4, serve as design rules for maximizing the probability of a particular isomer or combination of isomers. Future experiments involving different sticky parameters are necessary to validate these design rules.

Maximizing the yield of a particular isomer is interesting because the isomer might function as a "patchy" particle if one of the isotopes were functionalized differently from the other. For example, if the polystyrene spheres contained a linker group that could bind to other polystyrene particles at a certain temperature or $\mathrm{pH}$, then one could first make $\mathrm{P}_{1} \mathrm{~S}_{3}$ molecules with the polystyrene spheres on the short axis and, in a second step, assemble these molecules into "supra-molecular" structures by activating the linker groups. The molecules would bind preferentially along their short axes. Other types of directional interactions could be engineered simply by optimizing for different molecules. While our system is not ideally suited to producing large numbers of patchy particles, our experiments and models show that even in systems of particles with isotropic interactions, the structures of self-assembled molecules might be controlled to a surprisingly high extent through variables that are easily tuned in experiment.

Future studies might include cataloging the transition states in heterogeneous molecules and examining the structures and design rules for heterogeneous three-dimensional molecules. Using a combination of different sticky parameters should allow one to maximize the probability of specific types of non-rigid clusters. This could be useful for studying the hydrodynamics of diamondsquare-diamond ${ }^{28}$ and hinge-like modes. ${ }^{17}$ We used depletionbound silica and polystyrene microspheres in the experiments to inform our design criteria, but the model is not specific to this type of attractive interaction, nor to these particular isotopes. It applies also to DNA-mediated interactions, which should allow more control over the sticky parameters and number of interactions. ${ }^{27}$. Another intriguing direction would be to use the temperaturedependence of DNA-mediated interactions to dynamically switch the interaction strengths and modulate the distribution of isomers. 29

\section{Acknowledgments}

We thank Mike Cates for helpful discussions, Dave Kaz for designing the microscope stage modification, and Nica Franklin for helping us to formulate the experimental system. Rebecca W. Perry acknowledges the support of a National Science Foundation (NSF) Graduate Research Fellowship. This work was funded by the NSF through grant no. DMR-1306410 and by the Harvard
MRSEC through grant no. DMR-1420570.

\section{References}

1 A. van Blaaderen, Science, 2003, 301, 470-471.

2 V. N. Manoharan, M. T. Elsesser and D. J. Pine, Science, 2003, 301, 483-487.

3 D. J. Kraft, J. Groenewold and W. K. Kegel, Soft Matter, 2009, 5, 3823-3826.

4 A. Perro, E. Duguet, O. Lambert, J.-C. Taveau, E. BourgeatLami and S. Ravaine, Angewandte Chemie, 2009, 121, 367371.

5 E. Duguet, A. Désert, A. Perro and S. Ravaine, Chemical Society Reviews, 2011, 40, 941.

6 F. Li, D. P. Josephson and A. Stein, Angewandte Chemie International Edition, 2011, 50, 360-388.

7 Y. Wang, Y. Wang, D. R. Breed, V. N. Manoharan, L. Feng, A. D. Hollingsworth, M. Weck and D. J. Pine, Nature, 2012, 491, 51-55.

8 F. Ma, D. T. Wu and N. Wu, Journal of the American Chemical Society, 2013, 135, 7839-7842.

9 T. S. Skelhon, Y. Chen and S. A. F. Bon, Soft Matter, 2014, 10, 7730-7735.

10 G. Meng, N. Arkus, M. P. Brenner and V. N. Manoharan, Science, 2010, 327, 560-563.

11 F. Calvo, J. P. K. Doye and D. J. Wales, Nanoscale, 2012, 4, 1085-1100.

12 R. W. Perry, G. Meng, T. G. Dimiduk, J. Fung and V. N. Manoharan, Faraday Discussions, 2012, 159, 211-234.

13 E. Janai, A. B. Schofield and E. Sloutskin, Soft Matter, 2012, 8, 2924-2929.

14 J. W. R. Morgan and D. J. Wales, Nanoscale, 2014, 6, $10717-$ 10726.

15 D. Ortiz, K. L. Kohlstedt, T. D. Nguyen and S. C. Glotzer, Soft Matter, 2014, 10, 3541-3552.

16 R. S. Hoy, Physical Review E, 2015, 91, 012303.

17 R. W. Perry, M. C. Holmes-Cerfon, M. P. Brenner and V. N. Manoharan, Physical Review Letters, 2015, 114, 228301.

18 M. Cates, Self-Assembly and Entropy of Colloidal Clusters, http://www.condmatjournalclub.org/wpcontent/uploads/2012/12/JCCM_DECEMBER2012_ 01.pdf, 2012.

19 M. E. Cates and V. N. Manoharan, Soft Matter, 2015, 11, 6538-6546.

20 S. Sacanna, D. J. Pine and G.-R. Yi, Soft Matter, 2013, 9 , 8096-8106.

21 M. Grünwald and P. L. Geissler, ACS Nano, 2014, 8, 58915897.

22 H. N. W. Lekkerkerker and R. Tuinier, Colloids and the Depletion Interaction, Springer Science+Business Media B.V., Dordrecht, 2011.

23 P. D. Kaplan, L. P. Faucheux and A. J. Libchaber, Physical Review Letters, 1994, 73, 2793-2796.

24 D. B. Allan, T. A. Caswell and N. C. Keim, Trackpy $\quad$ 0.2 (DOI: $10.5281 /$ zenodo.9971), 
https://github.com/soft-matter/trackpy, 2014, http://dx.doi.org/10.5281/zenodo.9971.

25 J. C. Crocker and D. G. Grier, Journal of Colloid and Interface Science, 1996, 179, 298-310.

26 M. Holmes-Cerfon, S. J. Gortler and M. P. Brenner, Proceedings of the National Academy of Sciences, 2013, 110, E5-E14.

27 N. Geerts and E. Eiser, Soft Matter, 2010, 6, 4647.

28 W. N. Lipscomb, Science, 1966, 153, 373-378.

29 W. B. Rogers and V. N. Manoharan, Science, 2015, 347, 639642. 


\title{
Electronic Supplementary Material for Isotope Effects in Colloidal Molecules
}

\author{
Rebecca W. Perry ${ }^{1}$ and Vinothan N. Manoharan ${ }^{1,2, *}$ \\ ${ }^{1}$ Harvard John A. Paulson School of Engineering and Applied Sciences, \\ Harvard University, Cambridge, Massachusetts 02138, USA \\ ${ }^{2}$ Department of Physics, Harvard University, \\ Cambridge, Massachusetts 02138, USA
}

(Dated: January 24, 2016)

\section{SAMPLE PREPARATION PROTOCOL}

We used the following protocol to construct sample chambers for the self-assembly of $2 \mathrm{D}$ colloidal molecules.

1. Prepare one small $(22 \mathrm{~mm} \times 22 \mathrm{~mm})$ and one large $(24 \mathrm{~mm} \times 60 \mathrm{~mm})$ glass coverslip (VWR Micro Cover Glasses, No. 1) by rinsing with deionized water and drying with high-purity compressed nitrogen.

2. Plasma clean the large coverslip for 2 minutes in a PDC-32G Plasma Cleaner/Sterilizer (Harrick Plasma) with the RF Level set to High. Plasma cleaning greatly reduces sticking between particles and the coverslip. Only the large coverslip needs to be plasma cleaned because it will form the bottom of the sample chamber and, thus, be the surface on which the 2D self-assembly occurs. We found that using coverslips instead of glass slides was essential for preventing unwanted particle sticking.

3. To assemble the sample chamber, center the small coverslip on the large coverslip and separate them with two narrow (approximately 3-mm-wide) strips of 35- $\mu \mathrm{m}$-thick Mylar ${ }^{\circledR}$ A film (wiped clean with isopropanol) parallel to the long edges of the large coverslip. With the two coverslips clamped together (e.g., with binder clips), use UV-curing Norland Optical Adhesive 61 and a UV lamp to seal the two edges of the small coverslip parallel to the spacers. We find that sealing the four corners and then removing the clips before sealing the two edges works well.

4. Use a pipette to dispense well-dispersed colloidal suspension near one of the two unsealed edges of the small coverslip and let capillary action fill the sample chamber.

*vnm@seas.harvard.edu 
5. Use Devcon 5 Minute ${ }^{\circledR}$ Epoxy to seal the two unsealed edges of the small coverslip and to go over the two previously sealed edges for extra protection against evaporation.

6. Keep the sample chamber oriented such that the large coverslip forms the bottom of the chamber. In this transparent chamber, self-assembly can be directly observed with an inverted microscope.

We thank Jerome Fung for teaching us how to make this style of sample chamber.

\section{IMAGE PROCESSING}

Our custom image processing routine identifies isomers after the individual particles have been located with Trackpy[1]. Here, we describe our post-processing routine on the data used in this study.

The first step is to identify which of the 115,825 found particles are polystyrene, which are silica, and which are false positives. To do this, we plot the sizes of the particles returned by Trackpy (that is, the radii of the bright spots shown in Figure 2 of the manuscript, which are not the true particle radii) versus the particles' intensity maxima in the raw images. By plotting the data in this way, we see three distinct regions corresponding to silica particles, (27\%), polystyrene particles (40\%), and false positives (33\%) (Figure S1). By eye, we choose lines with slopes of 0.033 pixels/intensity value to delineate the three regions (Figure S1). We checked the lines' ability to discriminate particle types by examining the complete set of micrographs annotated with the determined particle types. We saw that more than $99 \%$ of the particles identified as silica or polystyrene were identified correctly. The main failure mode was silica particles that were either erroneously discarded as false positives or never located in the first place. Around $15 \%$ of small molecules contained more particles than were identified by the algorithm. Less than $1 \%$ of molecules had fewer particles than the algorithm determined. Wecorrected misidentifications in our list of molecules manually.

Next, we use proximity to group the individual particles into molecules. We calculate the distances between all possible pairs of particles in an image and, for each particle, we make a list of the other particles within a "molecule-sized" search radius, which we set to $3.5 \mu \mathrm{m}$. This distance is sufficiently large to encompass all rigid and almost all non-rigid configurations of molecules with up to five 1-pm-diameter particles. We then use these sets of nearby particles to assign each particle to a molecule, which we label numerically. The list of each particle's associated molecule is then converted to a list of molecules including information about which particles are members of each molecule. This method of grouping particles into molecules has two failure modes. First, two small molecules will get grouped together if they lie entirely within the same search radius. We check for and manually correct such occurrences by looking at the micrographs annotated with the algorithmic results. Second, the search radius can truncate large molecules or encapsulate pieces of multiple nearby molecules. Such occurrences are flagged by our algorithm, and the particles in these molecules are removed from further analyses $(19 \%$ of the polystyrene and silica particles). We then eliminate $6 \%$ of the remaining particles 


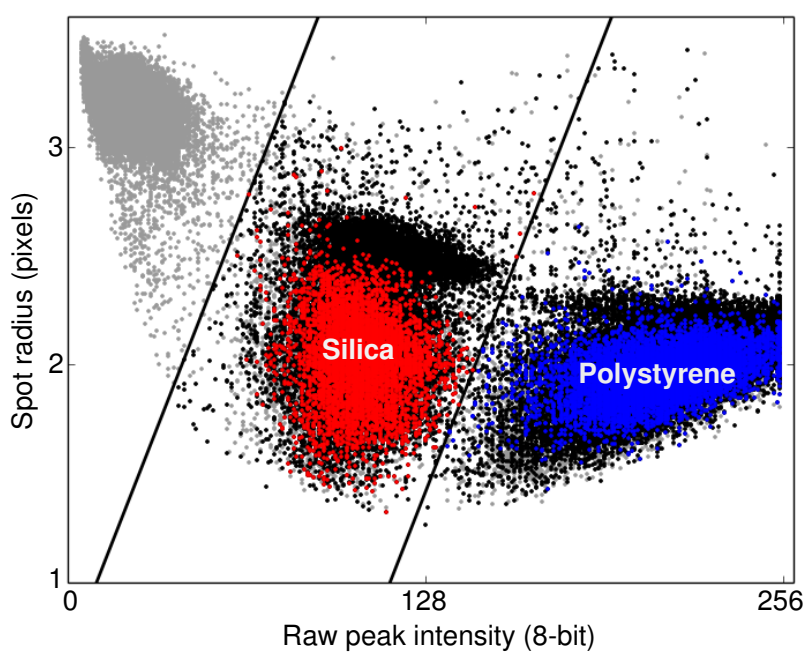

FIG. S1. Automatically distinguishing silica and polystyrene particles. A scatter plot of particle attributes reveals found "particles" of three types: silica particles (center), polystyrene particles (lower right), and false positives (upper left). The red and blue points are the particles in rigid 4- and 5-particle molecules. The clusters of black points directly above the colored regions are single particles of each isotope that are not bound to any other particles.

on the basis that the center of mass of each of their molecules is located less than 20 pixels $(2.3 \mu \mathrm{m})$ from the edge of an image. We do this to avoid analyzing molecules that are truncated by an edge.

To identify rigid molecules from the set of all molecules, we analyze the interparticle distances. To classify pairs of particles as bound or unbound we set a cutoff distance of $1.18 \mu \mathrm{m}$, about $15 \%$ larger than the distance between two bonded particles. Molecules with interparticle distances slightly larger than a particle diameter are likely to have been in the neighboring rigid state just after or just prior to the instants their images were captured. Our cutoff distance effectively rounds molecules with slight bond breaks to their nearest rigid molecules and also allows for some polydispersity among the particles.

Finally, we determine each rigid 4- and 5-particle molecule's configuration in terms of the set of possible isomers. We use a modified adjacency matrix that encodes the locations of different isotopes within a molecule. In previous studies on homogeneous molecules,[2-4] we used adjacency matrices to distinguish between molecules of different geometries. The standard adjacency matrix is populated with binary values, indicating whether the particles are separated (0) or bound (1). Here, to keep track of the different types of bonds, we use a 1 for an S-S bond, a 2 for an $\mathrm{P}-\mathrm{S}$ bond, and a 3 for a P-P bond (Figure S2). To obtain an isomer identification from the adjacency matrix, we perform a column sum and sort the resulting onedimensional list from low to high. This method yields a list that uniquely identifies each 4- and 5-particle isomer composed of 1 or 2 isotopes (note that enantiomers are grouped together). This approach to identifying isomers is not a general solution 

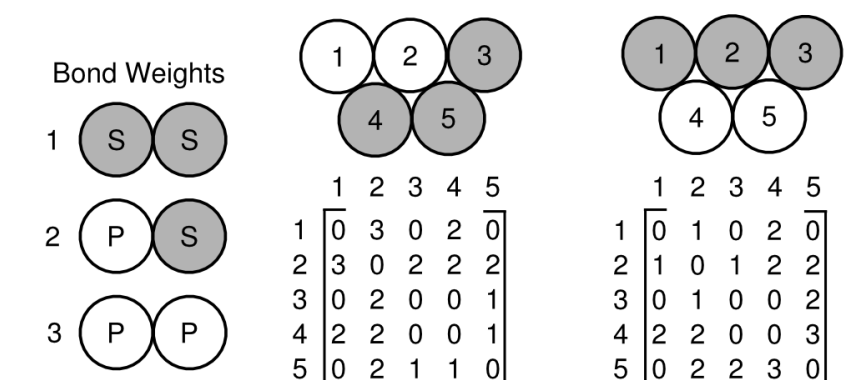

$\begin{array}{lllll}1 & 2 & 3 & 4 & 5\end{array}$

$1\left[\begin{array}{lllll}0 & 3 & 0 & 2 & 0\end{array}\right.$

$2 \quad \begin{array}{lllll}3 & 0 & 2 & 2 & 2\end{array}$

$\begin{array}{llllll}3 & 0 & 2 & 0 & 0 & 1\end{array}$

$4 \begin{array}{lllll}2 & 2 & 0 & 0 & 1\end{array}$

$5\left[\begin{array}{lllll}0 & 2 & 1 & 1 & 0\end{array}\right.$

Column sum:

$\begin{array}{lllll}5 & 9 & 3 & 5 & 4\end{array}$

$\begin{array}{lllll}1 & 2 & 3 & 4 & 5\end{array}$

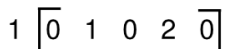

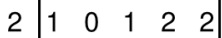

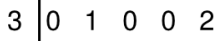

$4 \quad 2 \quad 2 \quad 00003$

$5\left[\begin{array}{lllll}0 & 2 & 2 & 3 & 0\end{array}\right.$

Sorted column sum:

$[3,4,5,5,9]$

$\begin{array}{lllll}3 & 6 & 3 & 7 & 7\end{array}$

$[3,3,6,7,7]$

FIG. S2. Modified adjacency matrix for colloidal molecules with two species. These two 5 -particle molecules have identical compositions and identical numbers of bonds of each type (S-S, P-S, P-P), yet are distinct isomers. A modified adjacency matrix with 1's, 2's, and 3's indicating different bond types is converted to a sorted column sum that is a unique identifier for all rigid one- and two-species isomers with fewer than 6 particles, regardless of the order in which the particles are numbered.

to labeling networks constructed from two types of nodes, but it is sufficient for our small molecules. We do not examine molecules with 6 or more particles because they have multiple rigid states and require a much larger data set to investigate their many isomers.

\section{INTERACTIVE VISUALIZATION OF CONTROLLABLE SELECTIVITY}

The interactive visualization accessible at http://people.seas.harvard.edu/ $\sim$ vnm/isotopes/clusters.html uses our model to allow users to modify two sticky parameters and explore the possibilities for selective placement of dopants.

[1] D. B. Allan, T. A. Caswell and N. C. Keim, Trackpy v0.2 (DOI: 10.5281/zenodo.9971), https://github.com/soft-matter/trackpy, 2014, http://dx.doi.org/ 10.5281/zenodo. 9971 .

[2] G. Meng, N. Arkus, M. P. Brenner and V. N. Manoharan, Science, 2010, 327, 560-563.

[3] R. W. Perry, G. Meng, T. G. Dimiduk, J. Fung and V. N. Manoharan, Faraday Discussions, 2012, 159, 211-234.

[4] R. W. Perry, M. C. Holmes-Cerfon, M. P. Brenner and V. N. Manoharan, Physical Review Letters, 2015, 114, 228301. 